\title{
Moving Mirrors and Thermodynamic Paradoxes
}

\author{
Adam D. Helfer \\ Department of Mathematics, University of Missouri - Columbia, Columbia, MO 65211, U.S.A.
}

(November 13, 2018)

\begin{abstract}
Quantum fields responding to "moving mirrors" have been predicted to give rise to thermodynamic paradoxes. I show here that the assumption in such work that the mirror can be treated as an external field is invalid, and the exotic energy-transfer effects necessary to the paradoxes are well below the scales at which the model is credible. A model with a first-quantized point-particle mirror is considered; for this it appears that exotic energy-transfers are lost in the quantum uncertainty in the mirror's state. Examining the physics giving rise to these limitations shows that an accurate accounting of these energies will require a model which recognizes the mirror's finite reflectivity, and almost certainly a model which allows for the excitation of internal mirror modes, that is, a second-quantized model.
\end{abstract}

\section{INTRODUCTION}

Almost twenty-five years ago, Davies and Fulling [1,2], following a suggestion of DeWitt [3] (see also [4]), introduced the "moving mirror" models: massless scalar quantum fields in two-dimensional Minkowski space responding to perfectly reflecting boundaries. Such models have been of inestimable value in clarifying conceptual issues raised by more complicated theories; most notably, there are connections between moving mirror models and the Hawking process.

There are still aspects of the moving-mirror models which are not satisfactorily understood. The most important of these are the thermodynamic paradoxes, which seem to be consequences of basic features of the models, and so unavoidable in them. Consider for simplicity the case of a non-relativistic mirror with position $q(t)$. Then, assuming the field was initially in the vacuum state (and that in the far past the mirror was stationary), the expected energy on the right (respectively, left) of the mirror is

$$
\left\langle E_{\text {right }}^{\text {left }}\right\rangle=\mp(12 \pi)^{-1}(\hbar / c) \ddot{q}+O\left(1 / c^{2}\right) .
$$

Now we come to the key point. It follows immediately that the total expected energy in the field is

$$
\left\langle E_{\text {left }}+E_{\text {right }}\right\rangle=0+O\left(1 / c^{2}\right),
$$

so (to lowest order) no energy is required to move the mirror. This is extraordinary. The leading effect of the mirror's motion is to separate the vacuum into packets of positive and negative expected energy, at no energetic cost. If these expectation-values can be regarded as classical energies, then we have a direct violation of the second law of thermodynamics. One can easily construct paradoxes based on this, and in fact Davies described a perpetual-motion machine which turns around this idea [5]. (See also [6].)

One might raise some objections to specific elements of Davies's proposal, and indeed various workers have done so (mainly concentrating on the problems of absorbing the negative energy packets $[7]$; see also the earlier paper [6]). Still, it seems hard to avoid the central point: if one can split the vacuum into positive- and negativeenergy parts, with negligible energetic input, isn't one violating the second law? Even setting aside possible difficulties associated with managing or absorbing the negative-energy packets, couldn't one drive perpetualmotion machines by simply keeping the positive energies produced by the mirror's motion, leaving the negativeenergy packets to go their ways?

The aim of the present paper is to resolve this point. I shall show that the approximation that has been made, that the mirror's trajectory can be treated classically, is invalid for the purpose of computing the necessary energy transfers. The limitations of validity of the classical model are reached before effects of energy-exchange between it and the quantum field can be computed. Thus, insofar as the mirror can be treated classically, there is no violation of the second law.

Going beyond this classical model, I shall consider a model with a non-relativistic, first-quantized mirror moving in an external potential. At least where the potential is quadratic, it will be shown that the measurement of the energy packets is always accompanied by a much larger spread in the mirror's energy. This means that attempts to measure the vacuum field energies cause quantum fluctuations in the mirror's state, fluctuations that cannot be ignored for the purposes of understanding the energy transfers between the field and the mirror.

It should be emphasized that the present work indicates that any "semiclassical" attempt to model the quantum back-reaction on the mirror is invalid for the purposes of modeling the energy-transfers that would occur in attempts to measure the vacuum field energy density. This is because semiclassical approximations (which give the back-reaction of the quantum field on the mirror in terms of expectations) are precisely those which assume that fluctuations in the mirror's state are negligible, and this is just what fails here. 
The present results fit well with those of a related investigation, by Parentani 10]. He introduced a model with a second-quantized mirror in a linear external potential. He was able to show (with certain approximations) that the forward quanta would decohere. This is because their states become correlated with that of the mirror. The general lesson to be drawn from the models, then, is that the entanglement of the mirror's state with that of the field can be a dominant effect, and the entanglement can involve fluctuations in the mirror's energy larger than the field's energy.

One can view the present work as estimating the magnitudes of the effects of quantum fluctuations in the mirror's state on the energy-transfers in the system; the effects are large enough to invalidate the external-field approximation. However, to go beyond this negative conclusion, and analyze in detail what does happen in the energy transfers, is another issue. I shall argue below that even this model is probably inadequate for a satisfactory understanding of these issues, and it will be necessary to pass to a theory where the internal degrees of freedom of the mirror (and the scattering of virtual field quanta from these) are accounted for. This is surprising and perhaps disconcerting: one would have thought that a quantum field responding to a slow, heavy mirror could be analyzed without needing to account for the mirror's structure as a system of quantum fields. But if one wants to understand the vacuum energies, such an analysis seems necessary.

Although such a sophisticated model will ultimately be necessary, there are good reasons for considering the non-relativistic first-quantized point-particle model, at least initially. The most important one is that there is little ambiguity in defining it, whereas to go beyond it requires many choices. (The more sophisticated models require one to make assumptions amounting to a choice of dispersive susceptibility $\chi(\omega)$, and there is functional freedom in doing so.) The non-relativistic mirror, by contrast, can be a first-quantized point particle, and there is little ambiguity in how to proceed. Thus the point-particle results, while more limited, are at least clearly model-independent.

Another reason for starting with the first-quantized point-particle model is that the quantum measurement issues can be analyzed at a fairly elementary level. Finally, the model is perhaps of some interest beyond the present paper. The very fact that it is of limited validity can be turned to advantage, because higher relativistic corrections can be ignored and a great deal of its structure can be worked out explicitly.

In Section II, the Davies-Fulling models are reviewed. This section may be read rapidly, but should not be skipped. No details of the calculations are given, but the physical basis of the renormalization and some of the limitations on the validity of the model are discussed in IIB. These limitations figure essentially in later arguments. Section III briefly derives the first-quantized mirror model. Section IV gives the main analysis of the measurement of field vacuum energy and its limitations. The last section summarizes the main conclusions.

In most places, particularly in estimates of the magnitudes of energies, factors of $c$ and $\hbar$ are given explicitly. However, factors of $c$ have been omitted in a few places (advanced and retarded coordinates, etc.), where they would make the appearance of the equations unnecessarily complicated.

\section{A CLASSICAL MOVING MIRROR}

In this section, I shall review the standard treatment of a massless field influenced by a moving mirror in twodimensional Minkowski space [2]. No details of standard computations will be given; the emphasis will be on the physical assumptions and consequences.

\section{A. Basic Formalism and Results}

Let $(t, x)$ be coordinates on two-dimensional Minkowski space with metric $d s^{2}=d t^{2}-d x^{2}$. We introduce retarded and advanced null coordinates by $u=t-x$ and $v=t+x$ as usual, and vectors $l^{a}=\partial_{v}, n^{a}=\partial_{u}$. It is convenient to regard the trajectory of the mirror as given by $v=V(u)$ or $u=U(v)$. We assume that the trajectory is timelike and is asymoptotically stationary in the past.

We consider a massless scalar field. Any solution to the field equation can be written locally as $\phi(u, v)=f(u)+$ $g(v)$. The mirror is considered to enforce the boundary condition $\phi(u, V(u))=0$. Thus we must have $f(u)=$ $-g(V(u))$. We shall write

$$
\phi=f(u)-f(U(v)), \quad v<V(u)
$$

(to the left of the mirror) and

$$
\phi=g(v)-g(V(u)), \quad v>V(u)
$$

(to the right). Thus the symbol $f$ will only be used for fields on the left, and $g$ only for fields on the right. Then the functions $f$ and $g$ can be considered data at $\mathcal{I}^{-}$for the field. We may also interpret these equations at the operator level; then $\hat{f}$ and $\hat{g}$ are the "in" operators.

The stress-energy operator is

$$
\widehat{T}_{a b}=: \widehat{T}_{a b}:+\left\langle\widehat{T}_{a b}^{\mathrm{ren}}\right\rangle
$$

where the colons stand for normal ordering and $\left\langle\widehat{T}_{a b}^{\mathrm{ren}}\right\rangle$ is the renormalized vacuum expectation value. This last is defined by point-splitting. One starts with the formal expression

$$
\widehat{T}_{a b}^{\text {formal }}=\left.\left.\left(\delta_{a}^{p} \delta_{b}^{q}-(1 / 2) g_{a b} g^{p q}\right) \nabla_{p} \hat{\phi}\right|_{\left(u_{1}, v_{1}\right)} \nabla_{q} \hat{\phi}\right|_{\left(u_{2}, v_{2}\right)}
$$


and considers the limit as $\left(u_{2}, v_{2}\right) \rightarrow\left(u_{1}, v_{1}\right)$. The expectation value $\left\langle 0\left|\widehat{T}_{a b}^{\text {formal }}\right| 0\right\rangle$ contains two terms: a divergent one which is independent of position, and a finite term. It is the finite term which is $\left\langle\widehat{T}_{a b}^{\mathrm{ren}}\right\rangle$. The divergent term, present even in Minkowski space, is the "unrenormalized stress-energy of the Minkowski vacuum." The result is

$$
\left\langle\widehat{T}_{a b}^{\mathrm{ren}}\right\rangle=(12 \pi)^{-1} \hbar\left(\frac{3}{4}\left(\frac{V^{\prime \prime}}{V^{\prime}}\right)^{2}-\frac{1}{2} \frac{V^{\prime \prime \prime}}{V^{\prime}}\right) l_{a} l_{b}
$$

on the right. (On the left, one has an expression of the same form, with $U$ replacing $V$ and $n_{a}$ replacing $l_{a}$.) In the limit of non-relativistic motion, with the trajectory given by $x=q(t)$, we have

$$
\left\langle\widehat{T}_{a b}^{\mathrm{ren}}\right\rangle=-(12 \pi)^{-1}\left(\hbar / c^{2}\right)\left((1+\dot{q}) \partial_{t}^{3} q+3 \ddot{q}^{2} \dot{q}\right) l_{a} l_{b}+\cdots
$$

(We have given as many terms as we shall need later.) This is to be evaluated at the time $t^{\prime}$ such that $\left(t-t^{\prime}, x-\right.$ $\left.q\left(t^{\prime}\right)\right)$ is null future-pointing.

From these formulas, one can derive expressions for the expected renormalized energy in the field to the left and the right of the mirror:

$$
\begin{aligned}
\left\langle E_{\text {right }}\right\rangle & =-(12 \pi)^{-1}\left(\hbar / c^{2}\right) \int_{q(t)}^{\infty}\left(\partial_{t}^{3} q+3 \ddot{q}^{2} \dot{q}\right) d x \\
& =-(12 \pi)^{-1}(\hbar / c) \int_{-\infty}^{t}\left(\partial_{t}^{3} q+3 \ddot{q}^{2} \dot{q}\right) d t^{\prime} \\
& =-(12 \pi)^{-1}(\hbar / c) \ddot{q}+\cdots .
\end{aligned}
$$

(An integration by parts can be used to justify discarding the second term when passing to the last line.) On the left of the mirror, one has

$$
E_{\mathrm{left}}=+(12 \pi)^{-1}(\hbar / c) \ddot{q}+\cdots
$$

Thus, to lowest order, no total expected energy is produced, but the mirror's motion effects a separation of the vacuum energy into positive and negative terms. The leading nontrivial contribution to the total expected energy in the field is of order $\dot{x}$ (that is, $v / c$ ) smaller; it is

$$
\begin{aligned}
E_{\text {total }} & =-(6 \pi)^{-1}\left(\hbar / c^{2}\right) \int_{-\infty}^{t} \partial_{t}^{3} q \dot{q} d t^{\prime} \\
& =-(6 \pi)^{-1}\left(\hbar / c^{2}\right)\left[\ddot{q} \dot{q}+\int_{-\infty}^{t}(\ddot{q})^{2} d t^{\prime}\right] .
\end{aligned}
$$

Thus the total energy put into the field must be positive, if the motion is asymptotically inertial.

\section{B. The Renormalization}

While all of the foregoing is standard, one must remember that we do not at present have a first-principles understanding of the infinite vacuum energy density and (therefore) of its renormalization. While the standard computation of $\left\langle\widehat{T}_{a b}^{\text {ren }}\right\rangle$ will be accepted here (within a regime of applicability to be discussed shortly), since the interpretation of this quantity is critical to the physics of the mirror, it is appropriate to discuss what has been done carefully. These points are important:

(a) The "operator part" of $\widehat{T}_{a b}$ - that is, the operator modulo the addition of c-number terms like $\left\langle\widehat{T}_{a b}^{\mathrm{ren}}\right\rangle$ - is determined by the equation of motion for the fields, and so is unambiguously defined irrespective of the renormalization. In other words, different choices of renormalization can only affect the c-number terms.

(b) It is not trivial that the theory is renormalizable. The idealized perfect-reflector nature of the boundary causes a great deal of cancellation of ultraviolet contributions to the stress-energy in the neighborhood of the mirror. In a more realistic model, one would expect dispersive effects to disturb these cancellations. This would lead to terms which were formally divergent as one approached the mirror (although the theory itself would break down as one approached within a distance of the order of the skin depth of the mirror). Cf. ref. [11].

A related point is that we have ignored whatever internal physics the mirror has which causes it to reflect. For an actual (electromagnetic) mirror, there are ions and conduction electrons whose contributions to the electromagnetic stress-energy outside the mirror might not be ignorable.

(c) Consider for the moment replacing the perfectly reflecting mirror by a more realistic model, where one has a mirror with a dispersive susceptibility tending rapidly to zero beyond some cut-off frequency $\omega_{\mathrm{p}}$. The effect of this would be to introduce a frequency-dependent potential term into the equation of motion, or equivalently, in coordinate space, a convolution of $\hat{\phi}$ with the Fourier transform of that potential. This term would act like a perfect reflector on field modes of frequencies $\omega \ll \omega_{\mathrm{p}}$, but the structure of the potential would become important at scales $\omega \sim \omega_{\mathrm{p}}$.

In such a model, the mirror will act like a classical reflector of low-frequency modes only as long as the time scale defined by its acceleration is significantly larger than $1 / \omega_{\mathrm{p}}$. If the acceleration is greater, we must take into account the mixing of low-frequency and highfrequency modes due to the mirror's motion.

What this means for the present paper is that the computation of $\left\langle\widehat{T}_{a b}^{\mathrm{ren}}\right\rangle$ is only credible as long as the inverse time scales over which $\dot{q}$ changes are much less that the plasma frequency $\omega_{\mathrm{p}}$ of the mirror. In particular, we must have $|\ddot{q}| \lesssim \omega_{\mathrm{p}}|\dot{q}|$ or we are not justified in using the standard formula, equation (7), and its consequences, equations (8)-(14).

(d) The usual procedure is to take the points $\left(u_{1}, v_{1}\right)$ and $\left(u_{2}, v_{2}\right)$ separated by a small imaginary timelike interval. This has the effect of introducing an ultraviolet cut-off. This is attractive, because one can then argue 
that the justification of the procedure is that real experiments only probe an object up to a finite frequency. Also this procedure ascribes to Minkowski space-time a (divergent) positive expected energy density, whereas realseparated points give rise to negative energy densities.

However, this procedure requires one to consider the world-line $V(u+i \delta u)$ at complex points as well, and it is hard to give a physical interpretation of this. If $V$ is analytic, of course, one has a clear candidate definition for $V(u+i \delta u)$. However, even in this case $V(u+i \delta u)$ depends non-locally on the real trajectory $V(u)$. It is in particular hard to see how to reconcile one's notions of causality (being able to change $V(u)$ freely in the future of $u=u_{0}$, irrespective of its behavior in the past) with the requirement of analyticity.

In practice, this point is usually ignored, and $V(u+i \delta u)$ simply represented by a Taylor series whose convergence is not questioned. We remark that if $V(u)$ is not analytic but Cauchy's formula is used to provide a candidate definition for $V(u+i \delta u)$, the c-number term $\left\langle\widehat{T}_{a b}^{\mathrm{ren}}\right\rangle$ becomes divergent; the theory is not renormalizable.

We shall not pursue this question of how or whether the standard renormalization is justified. Still, it is a point which is not really satisfatorily understood.

\section{A FIRST-QUANTIZED MIRROR}

In order to estimate the effects of quantum fluctuations in the state of the mirror on the energy exchanges between it and the field, we must quantize the mirror. We shall consider a simple model, in which the mirror is considered to be heavy and its motion non-relativistic. Then the mirror may be treated as a first-quantized particle. Let us begin by anticipating the limitations of this model.

(a) If the mirror's mass is $m$, then the model will only be valid for field modes of frequencies $\ll m c^{2} / \hbar$. The mass provides an effective ultraviolet cut-off.

(b) The model can accurately predict dynamics only for a finite time. This is because eventually relativistic corrections to phases become significant. Correspondingly, there will be a limit to the accuracy of the energy levels predicted by the model.

(c) It will be most important to recall that a firstquantized model is only valid at length scales greater than the Compton wavelength $\hbar /(m c)$. At smaller length scales, attempts to measure the position of the particle require localized energies large enough that pair creation (here, of quanta of the "mirror" field) becomes non-negligible, and this precisely means that the firstquantized model breaks down. This means that the position operator $q$ of the mirror only has a well-defined correspondence with physical reality on greater length scales.

(d) Even on the one-particle Hilbert space, relativistic corrections make the inner product $\langle\psi \mid \psi\rangle$ non-local with a length scale of order $\hbar /(m c)$. This means that, as far as the one-particle model makes sense, the quantum observable $q$ always has a spread of at least the order of the Compton wavelength.

The general strategy will be to first consider the mirror as classical and moving in a specified external potential $\mathcal{V}(q)$, and then promote the mirror's position $q$ and momentum $p$ to quantum operators. The Hamiltonian of the mirror is just $p^{2} /(2 m)+\mathcal{V}(q)$, so the main work involved is to compute the Hamiltonian of the field.

In fact, for the purposes of the present paper, it is only really necessary to compute the contributions to the vacuum energy part of the Hamiltonian: the normal-ordered terms are not needed. Still, we shall give these terms, for the purpose of making clear just what the model is. The dynamical consequences of the terms will be investigated elsewhere.

We begin by working out the contributions to the field Hamiltonian at $t=0$ from the left and the right of the mirror. (The choice $t=0$ is of course conventional; other choices of time slice will be related by unitary transformations.) We have

$$
\widehat{H}_{\substack{\text { right } \\ \text { left }}}=: \widehat{H}_{\text {right }}^{\text {left }}: \mp(12 \pi)^{-1} \ddot{q} .
$$

Using the mirror's equation of motion, we will replace $\ddot{q}$ by $-(1 / m) \mathcal{V}^{\prime}$. For the normal-ordered terms, we have

$$
: \widehat{H}_{\text {right }}:=\int_{q(0)}^{\infty}:\left(\hat{g}^{\prime}(-x)\right)^{2}+\left(V^{\prime}(-x) \hat{g}^{\prime}(V(-x))\right)^{2}: d x,
$$

with : $\widehat{H}_{\text {left }}$ : given by a similar expression. We now re-write the contribution from the second term, in two steps. We have

$$
\int_{q(0)}^{\infty}:\left(V^{\prime}(-x) \hat{g}^{\prime}(V(-x))\right)^{2}: d x=\int_{-\infty}^{q(0)}: \hat{g}^{\prime}(v)^{2}: V^{\prime} d v .
$$

Since $V^{\prime}$ is a perturbation of unity, we split off a term where $V^{\prime}$ is replaced by unity, and combine it with the first term in : $\widehat{H}_{\text {right }}$ : to give the Hamiltonian of a free field (in the presence of a fixed mirror) plus a perturbation, which is

$$
\begin{aligned}
\int_{-\infty}^{q(0)} & : \hat{g}^{\prime}(v)^{2}:\left(V^{\prime}-1\right) d v \\
& =2 \int_{-\infty}^{0}: \hat{g}^{\prime}(t+q(t))^{2}: \dot{q}(t) d t
\end{aligned}
$$

Thus we have

$$
: \widehat{H}_{\text {right }}:=: \widehat{H}_{\text {right, fixed }}:+: \widehat{H}_{\text {right, pert }}: \text {, }
$$

where 


$$
: \widehat{H}_{\text {right, fixed }}:=\int_{-\infty}^{\infty}: \hat{g}^{\prime}(x)^{2}: d x
$$

and

$$
: \widehat{H}_{\text {right, pert }}:=2 \int_{-\infty}^{0}: \hat{g}^{\prime}(t+q(t))^{2}: \dot{q}(t) d t .
$$

This term is already of order $v / c$. Thus we may compute $q(t)$ and $\dot{q}(t)=p(t) / m$ to the required accuracy from the mirror's Hamiltonian

$$
\widehat{H}_{\text {mirror }}=p^{2} /(2 m)+\mathcal{V} .
$$

Using this, choosing a Hermitian factor-ordering, and abusing notation by keeping the same symbol for the Hamiltonian with quantized $p$ and $q$, we find

$$
\begin{aligned}
& : \widehat{H}_{\text {right, pert }}:=m^{-1} \int_{-\infty}^{0} e^{i \widehat{H}_{\text {mirror }} t} \\
& \quad \times\left(: \hat{g}^{\prime}(t+q(0))^{2}: p(0)+p(0): \hat{g}^{\prime}(t+q(0))^{2}:\right) \\
& \quad \times e^{-i \widehat{H}_{\text {mirror }} t} d t .
\end{aligned}
$$

This is the final expression for the normal-ordered part of the correction to the free-field Hamiltonian in the model with first-quantized mirror. As mentioned above, we do not really need this explicit form in what follows, but present it for the purposes of defining the model.

Before analyzing how passage to this model affects the paradoxes of the classical mirror, a few comments about the model's structure are in order. One can regard this model as a perturbation of a stationary classical mirror, the perturbation parameter being $\mathrm{m}^{-1}$. Adopting this point of view, one can ask how the eigenstates of the classical mirror are affected by taking into account its finite mass. The integrals over the halfline in equation (23) will contain creation $\otimes$ creation and annihilation $\otimes$ annihilation operators, and these will result in a "dressing" of the states. In particular, the vacuum state will be dressed with two-particle contributions. The mirror, too, will be affected by the operators $p$ and $q$; the dressing will contribute states which in the unperturbed theory would be excited.

\section{MEASUREMENT OF THE VACUUM ENERGY}

We now take up the question of how well the vacuum energy on either side of the mirror can be measured, and to what extent those measurements are compatible with the treatment of the mirror as a classical object.

Throughout this section, we consider the measurement of $\widehat{H}_{\text {right }}$. This means a measurement is made of the field energy on the entire half-space to the right of the mirror. (Of course, this is for many purposes an idealization. In many cases, one would consider the energy-content over a fixed region of space, and restrict the mirror to be on one side of that. However, such analyses are cumbersome and will not be attempted here.) We also assume that the field is initially in the vacuum state.

\section{A. The Classical Model}

In this subsection, we shall assume that the mirror is in a state which can be well-modeled by a classical trajectory. Thus we may assume that at any time $t$ the mirror's position and momentum may be measured to classical accuracies $\Delta q$ and $\Delta p$ which are larger than the spreads in the corresponding quantum observables. Then there is a classical limit to the accuracy to which the mirror's energy is known:

$$
\Delta H_{\text {mirror }} \simeq \frac{p}{m} \Delta p+\mathcal{V}^{\prime}(q) \Delta q .
$$

In particular, the limit of the accuracy in the energy due to the classical uncertainty in position must satisfy

$$
\left|\Delta H_{\text {mirror }}\right| \gtrsim\left|\mathcal{V}^{\prime} \Delta q\right| \text {. }
$$

However, note that $\Delta q$ must be far larger than the mirror's Compton wavelength for the mirror to be in a classical state. Thus we have

$$
\left|\Delta H_{\text {mirror }}\right| \gg\left\langle\widehat{H}_{\text {right }}\right\rangle .
$$

In other words, to the extent that the classical model of the mirror's trajectory is credible, the lack of accuracy in knowledge of the mirror's energy must be far larger than the vacuum energy in the field. This means that while the mirror's motion splits the vacuum into energy packets of opposite signs, the uncertainty in the energetic cost of this separation is far larger than the magnitude of the separation itself. Thus there is no detectable violation of the second law.

Note too that this means an attempt to consider a term like $\left\langle\widehat{H}_{\text {right }}\right\rangle$ as a semiclassical contribution to the mirror's energy is misguided. It is not of itself wrong, but it is a correction far below the scale at which any classical treatment of the mirror is valid.

\section{B. The First-Quantized Model}

In the previous subsection, we saw that energies of the scale $\left\langle\widehat{H}_{\text {right }}\right\rangle$ were far below contributions which could be meaningfully treated by a classical model of the mirror. This of course suggests that we must pass to a quantized mirror to understand the energy-transfers between it and the field. I shall do so here, using the first-quantized model, but I shall not attempt a full analysis of the problem. This is partly because of technical difficulties in the first-quantized model (as I shall explain), but there is a deeper reason. 
We saw in the previous subsection that the mirror's Compton wavelength entered in limiting the validity of the classical model. This length is the scale at which a first-quantized treatment breaks down, so we may expect that even the first-quantized model will be inadequate. This is indeed the case, as will be discussed below. However, the analysis of the first-quantized mirror will uncover a new physical effect in the energetics, and so we take it up here.

We have $\left\langle\widehat{H}_{\text {right }}\right\rangle=(12 \pi m)^{-1} \mathcal{V}^{\prime}(q)$. This means a measurement of the vacuum energy is essentially a measurement of $q$. (A strictly linear potential $\mathcal{V}=$ const. $q$ is excluded for several reasons. The most important of these is that the corresponding classical trajectories would not obey the boundary conditions necessary for the derivation of the formulas for $\left\langle\widehat{T}_{a b}^{\mathrm{ren}}\right\rangle$.) A measurement of $q$ is always made with a quantum uncertainty, and insofar as the first-quantized model is valid the spread in the quantum observable must be larger than the Compton wavelength:

$$
\Delta q \gtrsim \hbar /(m c)
$$

Note that while the symbol used $(\Delta q)$ is the same as in the previous subsection, the meaning here is different. Here $\Delta q$ represents not just a lack of knowledge or of measurement resolution, but the spread of the components of the wave function with respect to the spectral resolution of the operator $q$.

The spread in the mirror's potential energy is

$$
\Delta \mathcal{V} \simeq \mathcal{V}^{\prime} \Delta q \gtrsim \mathcal{V}^{\prime}(\hbar /(m c))
$$

which is far larger than the vacuum energy. This suggests the relation

$$
\Delta E_{\text {mirror }} \gtrsim\left\langle\widehat{H}_{\text {right }}\right\rangle
$$

that is, the spread in the mirror's energy must be larger than the vacuum energy in the field. This would mean that the vacuum energy could not be usefully separated from the mirror's own energy, and indeed the vacuum energy would have to be considered as part of the constitutive energy of the mirror.

Of course, the relation (29) has not been established rigorously, because we have neglected possible cancellations between the spreads of the mirror's kinetic energy and its potential energy. A careful argument would seem to be technically very difficult, especially as we have made essentially no restriction on the potential. However, the physical conclusion - that the spread in the mirror's energy is large compared to the vacuum energy - seems suggestive eneough that it is worthwhile to raise as a generic possibility.

We can establish the relation (29) in the case of a mirror moving in a quadratic potential $\mathcal{V}=k q^{2} / 2$. In this case, the vacuum energy is $(12 \pi m)^{-1} k q$, so a measurement of this is precisely a measurement of $q$. A reliable measurement of this energy therefore requires a measurement with nominal value $\bar{q}$ and spread $\Delta q$ related by $\Delta q / \bar{q}<1$. Now, since $\left[\widehat{H}_{\text {mirror }}, q\right]=i \hbar p / m$ we must have $\Delta E \Delta q \gtrsim \hbar \bar{p} / m$. On th other hand, we have $\Delta p \gtrsim \hbar / \Delta q$ and thus $\bar{p} \gtrsim \hbar / \bar{q}$. Thus the mirror's energy spread

$$
\begin{aligned}
\Delta E & \gtrsim \hbar /(m \bar{q} \Delta q) \\
& \gtrsim \hbar /\left(m \bar{q}^{2}\right) \\
& \gtrsim(\hbar / m)(m c / \hbar)(1 / \bar{q}) \\
& \gtrsim(\hbar / m)\left(k \bar{q}^{2} / \hbar c\right)(1 / \bar{q}) \\
& =(\hbar / m c) k \bar{q}
\end{aligned}
$$

which is the vacuum energy.

\section{Limitation of the First-Quantized Model}

The treatment of the mirror as a first-quantized particle is only accurate within certain regimes. An important limitation is that a real mirror does not reflect all frequencies perfectly, but becomes transparent to sufficiently high modes.

To understand this, let $\omega_{\mathrm{p}}$ be a "plasma" frequency, giving a scale beyond which the mirror becomes essentially transparent. Associated with this is a "skin depth" $c / \omega_{\mathrm{p}}$ to which modes penetrate before being reflected. The mirror's position, as a reflecting surface, is not defined more accurately than this skin depth. This means that the model is only credible insofar as it depends on spatial resolutions $\gtrsim c / \omega_{\mathrm{p}}$. Now for a realistic mirror we must have

$$
\hbar \omega_{\mathrm{p}} \ll m c^{2},
$$

that is, the plasma energy should be less than the rest energy, or equivalently, the skin depth should be much larger than the Compton wavelength. However, the vacuum energy on one side of the field is

$$
\left|(12 \pi)^{-1} \mathcal{V}^{\prime} \frac{\hbar}{m c}\right| \approx(12 \pi)^{-1}\left|\mathcal{V}\left(q+\frac{\hbar}{m c}\right)-\mathcal{V}(q)\right|
$$

is a measurement of the difference of potential energies over the Compton wavelength $\hbar /(m c) \ll c / \omega_{\mathrm{p}}$. Thus this energy difference is well below the ambiguity in the mirror's Hamiltonian

$$
\widehat{H}_{\text {mirror }}=\frac{p^{2}}{2 m}+\mathcal{V}
$$

We see that the first-quantized model is not accurate enough to determine whether there are exchanges of energy between the mirror and the field of the same scale as the vacuum energy. The limitation is in the treatment of the mirror as perfectly reflecting, and the neglect of whatever internal physics of the mirror gives rise to that refelection. Presumably, an accurate model will require passing beyond this. 


\section{SUMMARY AND IMPLICATIONS}

\section{A. Summary}

I have re-examined the "moving mirror" models of Davies and Fulling, giving attention to their limits of validity in computing energy transfers between the mirror and the vacuum energy of the field. Insofar as the mirror can be modeled by a classical point particle, we find that the lack of accuracy in its energy far exceeds the vacuum energy. This means that, while the motion of the mirror splits the vacuum into positive and negative energy packets, the magnitudes of those energies are far below the uncertainty in the mirror's energy. Thus no violation of the second law arises.

Moving beyond the classical model to first-quantized mirror, we were able to show, at least in the case of a quadratic external potential, that the quantum spread in the mirror's energy must be greater than the field's vacuum energy. This would mean that a measurement of the field energy would necessarily drive the mirror into a superposition of energy states, with width greater than the vacuum energy. For more general potentials, we gave suggestive but not conclusive arguments for the same conclusion.

These conclusions are consonant with the results of Parentani [10]. With a different although related purpose, he investigated a second-quantized mirror model with certain approximations, and found correlations between the mirror state and those of forward-scattered quanta. Taken together, the two models show: (a) that when vacuum field energies are measured, the entanglement of the mirror's state with that of the field may be a dominant effect; and (b) the entanglement may involve fluctuations in the mirror energy grater than the field's vacuum energy.

Even the model of the mirror by first-quantized point particle turned out to be insufficiently accurate for quantitative analysis of the energy transfers between the mirror and the field. We found that in order to reliably compute mirror energies to a resolution of the order of the vacuum energy, one will need to take into account the finite reflectivity of the mirror, and its structure on scales of the order of its skin depth.

We found too evidence for another limitation on energy measurements, deeper than that set by the finite reflectivity. At every point where we used the skin depth to restrict the limits of measurability of energies, we also used the Compton wavelength $\hbar /(m c) \ll c / \omega_{\mathrm{p}}$ of the mirror. The Compton wavelength is the scale at which the firstquantized, non-relativistic model of a particle (here, the mirror) breaks down (irrespective of its reflective properties). The appearance of this scale seems to indicate that in a deep way we must confront the infinitely many degrees of freedom of the mirror as a second-quantized object, before we will be able to have a satisfactory understanding of the transfers of energy between it and the field.

These requirements to pass to a very deep model of the mirror in order to reliably study the energetics of the system must be considered a surprise, because for a long time it has been assumed that at least for sufficiently heavy mirrors a classical model should be valid. However, we see that not only does this fail, but even a first-quantized mirror model is not sufficiently refined to make positive predictions.

\section{B. Implications}

These results have serious implications for attempts to understand the back-reaction of the quantum field on the mirror. Even the first conclusion, that such backreaction effects are below the range of applicability of the classical model, is important. It shows that any attempt to treat these back-reaction effects semiclassically is misguided, because the back-reactions are far smaller than the scales at which the classical model can be trusted anyway.

The second result (that the spread in the mirror's energy must be greater than the vacuum energy) shows that the semiclassical model is not merely insufficiently refined: its basic assumption is wholly misdirected. A semiclassical approximation precisely assumes that the quantum fluctuations in the mirror's state are negligible: but the opposite is the case.

It is hoped that the features uncovered in this simple model will be guides to the analysis of more complicated and realistic field theories. In particular, a main motivation for this work was as a warm-up for an analysis of similar issues in the Hawking process, where a wholly convincing understanding of the backreaction has yet to be reached.

[1] P. C. W. Davies and S. A. Fulling, Proc. R. Soc. Lond A356, 237 (1977).

[2] S. A. Fulling and P. C. W. Davies, Proc. R. Soc. Lond. A348, 393 (1976).

[3] B. S. DeWitt, Phys. Rep. 19C, 295 (1975).

[4] G. T. Moore, J. Math. Phys. 11, 2679 (1970).

[5] P. C. W. Davies, Phys. Lett. 113B, 215 (1982).

[6] L. H. Ford, Proc. R. Soc. Lond. A364, 227 (1978).

[7] P. G. Grove, Class. Quant. Grav. 3, 801 (1986).

[8] A. D. Helfer, Class. Quant. Grav. 15, 1169 (1998).

[9] A. D. Helfer, Mod. Phys. Lett. A13, 1637 (1998).

[10] R. Parentani, Nucl. Phys. B465, 4526 (1996).

[11] A. D. Helfer and A. S. I. D. Lang, J. Phys. A32, 1937 (1999). 